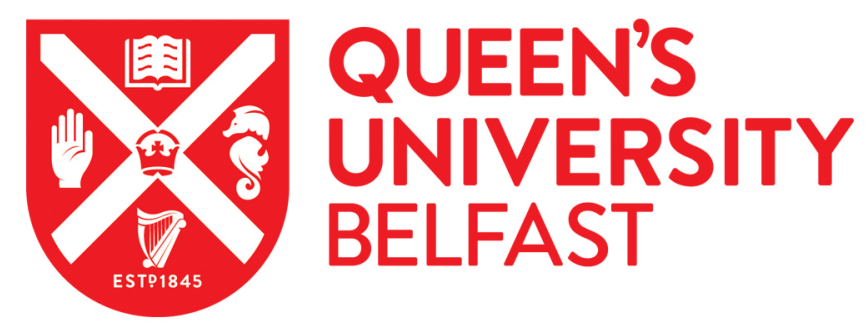

\title{
Doping-Driven Wettability of Two-Dimensional Materials A Multiscale Theory
}

Tian, T., Lin, S., Li, S., Zhao, L., Santos, E. J. G., \& Shih, C-J. (2017). Doping-Driven Wettability of TwoDimensional Materials A Multiscale Theory. Langmuir, 33(44), 12827-12837.

https://doi.org/10.1021/acs.langmuir.7b03165

\section{Published in:}

Langmuir

\section{Document Version:}

Peer reviewed version

Queen's University Belfast - Research Portal:

Link to publication record in Queen's University Belfast Research Portal

\section{Publisher rights}

(c) 2017 American Chemical Society. This work is made available online in accordance with the publisher's policies. Please refer to any applicable terms of use of the publisher.

\section{General rights}

Copyright for the publications made accessible via the Queen's University Belfast Research Portal is retained by the author(s) and / or other copyright owners and it is a condition of accessing these publications that users recognise and abide by the legal requirements associated with these rights.

Take down policy

The Research Portal is Queen's institutional repository that provides access to Queen's research output. Every effort has been made to ensure that content in the Research Portal does not infringe any person's rights, or applicable UK laws. If you discover content in the Research Portal that you believe breaches copyright or violates any law, please contact openaccess@qub.ac.uk. 


\title{
Multiscale Understanding the Wettability of Doped Two-Dimensional Materials
}

\author{
Tian Tian, ${ }^{\dagger}$ Siyu Li, ${ }^{\ddagger}$ Lingling Zhao ${ }^{\ddagger}$ Elton J. G. Santos, ${ }^{\top}$ Shangchao Lin, ${ }^{*}, \|$ \\ and Chih-Jen Shih*, $\uparrow$
}

$\dagger$ Institute for Chemical and Bioengineering, ETH Zürich, Vladimir Prelog Weg 1, CH-8093

Zürich, Switzerland

$\ddagger$ Key Laboratory of Energy Thermal Conversion and Control of Ministry of Education, School of Energy and Environment, Southeast University, Nanjing, Jiangsu 210096, China ISchool of Mathematics and Physics, Queen's University Belfast, United Kingdom $\S$ School of Chemistry and Chemical Engineering, Queen's University Belfast, United Kingdom

||Department of Mechanical Engineering, Materials Science and Engineering Program, FAMU-FSU College of Engineering, Florida State University, Tallahassee, Florida 32310, United States

E-mail: slin@eng.fsu.edu.; chih-jen.shih@chem.ethz.ch 


\begin{abstract}
Engineering molecular interactions at two-dimensional (2D) materials interfaces enables new technological opportunities in functional surfaces and molecular epitaxy. Quantifying the interplay between the interfacial forces and electric potential of 2D materials represents the crucial first step for understanding their wettability. Here we develop the first theoretical framework to model the wettability of the doped 2D materials by properly bridging the multiscale physical phenomena at the $2 \mathrm{D}$ interfaces, including: (i) the change of 2D materials surface energy (atomistic scale), (ii) the molecular reorientation of liquid molecules adjacent to the interface, (molecular scale), and (iii) the electrical double layer (EDL) formed in the liquid phase (macroscopic scale). The latter two effects are found to be the major mechanisms responsible for the contact angle change upon doping. When the doping level is electrostatically tuned, we demonstrate that 2D materials with high quantum capacitances (e.g., transition metal dichalcogenides, TMDCs) possess a wider range of tunability in the interfacial tension. Furthermore, practical considerations such as defect and airborne contamination are also quantitatively discussed. Our analysis implies that the doping level can be another variable to modulate the wettability at the interface of $2 \mathrm{D}$ materials, as well as the molecular packing behavior on a 2D material-coated surface, essentially facilitating the interfacial engineering of 2D materials.
\end{abstract}




\section{Introduction}

Two-dimensional (2D) materials, the crystalline films with a thickness of one atom or one molecule, combine optical transparency and the quantum-confined properties. ${ }^{1-3}$ Recent progress in the scalable production of large-area $2 \mathrm{D}$ materials ${ }^{4,5}$ has paved the way for the applications of functional surfaces and ultrathin membranes. ${ }^{6-11}$ The concept of van der Waals (vdW) epitaxy has been further extended to grow molecular heterostructures on 2D material-coated surfaces for a variety of applications. ${ }^{3,12,13}$ The realization of these technologies essentially requires precise control over the molecular interactions at 2D material interfaces, which motivates fundamental research of their interfacial properties, in particular the wettability. An accurate determination of the interfacial tension, nevertheless, remains highly disputed. ${ }^{14-17}$ Early literature on understanding the surface science of 2D materials has highlighted the effects of the airborne contaminants ${ }^{15,18-21}$ and the underlying substrate, ${ }^{22-25}$ since the length scale for the van der Waals (vdW) radius is comparable to the thickness of a monolayer. On the other hand, a subtle but important fact is that the 2D semimetals (e.g, graphene and silicene) and 2D semiconductors (e.g., transition metal dichalcogenides (TMDCs)) possess low density of states (DOS) around the intrinsic Fermi level $\left(E_{\mathrm{F}}\right)$, such that the effect of doping, either induced by the surroundings, ${ }^{26-28}$ or by the electrostatic gating, ${ }^{29,30}$ also comes into play. Recent advance in the ab initio calculations and scanning tunneling microscopy (STM) studies on the adsorbed molecules on graphene ${ }^{31,32}$ has provided some clues to the doping-dependent vdW interactions. Subsequently, the doping-induced change in the wettability of graphene has been observed in the contact-angle experiments, ${ }^{33-35}$ and the molecular dynamics (MD) simulations. ${ }^{36-39}$ In this respect, however, most reports described the doping-dependent wettability with the basic Young-Lippmann equation (YLE), ${ }^{40}$ whereas the interplay between the orientation of liquid molecules at the interface ${ }^{41}$ and the electronic structure of the $2 \mathrm{D}$ materials is often ignored. In order to address the discrepancies among the literature, a general and complete theoretical picture that bridges the gap between different length scales, is clearly required. 
In this report, we propose the first theoretical framework to model the change of interfacial tension between liquid and a sheet of monolayer 2D material. Multiscale physical phenomena are considered, as shown in Figure 1. At the atomistic scale, we formulate the dependence of surface energy of a 2D material on the doping density using the quantum capacitance (QC) calculated by the density functional theory (DFT). Next, the surfacecharge-induced reorientation of liquid molecules adjacent to the interface is associated with an N-body system, which is resolved by molecular dynamics (MD) simulations, allowing quantification of the interfacial tension in the absence of electrolytes in liquid. The effect of the electrical double layer (EDL) induced by the electrostatic interactions between the 2D material surface charges and the ionic species in liquid, is then addressed at the continuum level. Practical considerations, such as the defect density and the surface contamination are also taken into account to provide a comprehensive understanding of the phenomena. Finally, we examine and validate our theory by comparing with the contact angle changes reported in the electrowetting and the substrate-induced doping experiments.

\section{Results and Discussions}

\section{Surface Energy of Doped 2D Materials}

Consider a liquid $(L)$ droplet sitting on a flat, monolayer 2D material (2D) supported by a solid substrate, following the Young's equation, the change of the equilibrium contact angle $\theta$ upon doping, is given by

$$
\gamma_{\mathrm{L}} \Delta \cos \theta=\Delta \gamma_{2 \mathrm{D}}-\Delta \gamma_{2 \mathrm{D}-\mathrm{L}}
$$

where $\gamma_{\mathrm{L}}$ and $\gamma_{2 \mathrm{D}}$ are the surface tensions of liquid and the $2 \mathrm{D}$ material considered, respectively, $\gamma_{2 \mathrm{D}-\mathrm{L}}$ is the interfacial tension between the liquid and the contacting 2D material. We define $\Delta \gamma=\gamma-\gamma_{0}$ for all the surface (interfacial) tensions considered and 
$\Delta \cos \theta=\cos \theta-\cos \theta_{0}$, where the subscript 0 corresponds to that in the case of intrinsic $2 \mathrm{D}$ material, with the doping density per unit area, $\sigma_{2 \mathrm{D}}=0$. In the theoretical analysis presented here, we aim to model $\Delta \cos \theta$ as a function of $\sigma_{2 \mathrm{D}}$. Note that under the assumptions that (i) the change of $E_{\mathrm{F}}$ in the doped 2D materials does not result in the interfacial electron transfer, namely, the electrochemical reactions, (ii) the doping effect does not change the surface energy of the underlying substrate, and (iii) the vdW and electrostatic interactions are perfectly additive and pairwise, one can decouple the effect of the underlying substrate. Therefore the debate of the "wetting transparency", ${ }^{23,25}$ does not affect our analysis.

First, we model the dependence of $\gamma_{2 \mathrm{D}}$ on the doping level. Consider a closed system with constant pressure and volume containing a sheet of free-standing 2D material, following the Euler homogeneous function theorem of thermodynamics, the total internal energy of the system $U$, is given by $U=T S+\mu_{2 \mathrm{D}} N+\gamma_{2 \mathrm{D}} A+\psi_{2 \mathrm{D}} q,{ }^{42}$ where $T$ is the temperature, $S$ is the entropy, $\mu_{2 \mathrm{D}}$ is the chemical potential of the $2 \mathrm{D}$ material per unit lattice, $N$ is the number of unit lattices, $A$ is the area of the $2 \mathrm{D}$ material, and $\psi_{2 \mathrm{D}}$ and $q$ are the electric potential and total charge in the $2 \mathrm{D}$ material, respectively. At constant $T$, combining with the first law of thermodynamics and the differential form of $U$, it follows:

$$
\mathrm{d} \gamma_{2 \mathrm{D}}=-\frac{q}{A} \mathrm{~d} \psi_{2 \mathrm{D}}=-\sigma_{2 \mathrm{D}} \mathrm{d} \psi_{2 \mathrm{D}}
$$

relating the surface tension change of a free-standing 2D material as a function of its doping density $\sigma_{2 \mathrm{D}}$. After bringing an amount of charge $q$ from infinity to a charge-neutral 2D material in the aforementioned system, the surface energy change is therefore given by:

$$
\Delta \gamma_{2 \mathrm{D}}=-\int_{0}^{\psi_{2 \mathrm{D}}} \sigma_{2 \mathrm{D}} \mathrm{d} \psi^{\prime}=-\int_{0}^{\sigma_{2 \mathrm{D}}} \sigma^{\prime}\left(\frac{1}{C_{2 \mathrm{D}}}\right) \mathrm{d} \sigma^{\prime}
$$

where $C_{2 \mathrm{D}}=g\left(E_{\mathrm{F}}\right) e^{2}$ is the quantum capacitance of the $2 \mathrm{D}$ material, ${ }^{43,44} g\left(E_{\mathrm{F}}\right)$ is the DOS as a function of $E_{\mathrm{F}}, \psi_{2 \mathrm{D}}=-\left(E_{\mathrm{F}}-E_{\mathrm{F}, 0}\right) / e$ and $E_{\mathrm{F}, 0}$ corresponds to the Fermi level of the 2D material at the charge neutral point (CNP). Accordingly, eq 3 provides a simple relation 
which depicts the surface tension change of a 2D material at the quantum-mechanical level. We have calculated the DOS as a function of $E_{\mathrm{F}}$ for a variety of $2 \mathrm{D}$ materials using the density functional theory. ${ }^{45}$ Note that we take into account a fractional component of the exact exchange from the Hartree-Fock (HF) theory hybridized with the DFT exchangecorrelation functional at the level of the HSE06 hybrid functional. Therefore, any limitation of the exchange and correlation functional utilized in the chemical description of the energy levels can be improved. The following 2D materials are considered: TMDC monolayers $\left(\mathrm{MX}_{2}, \mathrm{M}=\mathrm{Mo}, \mathrm{W}\right.$ and $\left.\mathrm{X}=\mathrm{S}, \mathrm{Se}, \mathrm{Te}\right)$, silicene, germanene, phosphorene (monolayer black phosphorus), and graphene. The doping density in a 2D material is calculated by integrating the DOS from its intrinsic Fermi level, i.e., $\sigma_{2 \mathrm{D}}=\int_{E_{\mathrm{F}, 0}}^{E_{\mathrm{F}}} g\left(E^{\prime}\right) e \mathrm{~d} E^{\prime}{ }^{46}$

Figure 2 presents the calculated $\Delta \gamma_{2 \mathrm{D}}$ as a function of $\sigma_{2 \mathrm{D}}$ for the 2D materials considered here. Clearly, the doping of 2D materials reduces their surface energy, or based on the classical definition, the work required to separate two stacked monolayers is lowered. Among the $2 \mathrm{D}$ materials, we find that graphene shows the highest degree of surface energy decrease, up to $-16 \mathrm{~mJ} \cdot \mathrm{m}^{-2}$, or $\sim 20 \%$ reduction of its intrinsic surface tension, ${ }^{25}$ at $\sigma_{2 \mathrm{D}}= \pm 4 \times 10^{13} \mathrm{e}$. $\mathrm{cm}^{-2}$. However, as will be discussed later, it does not imply a reduced wettability because the quantum capacitance effect also reduces the interfacial tension, $\gamma_{2 \mathrm{D}-\mathrm{L}}$. A clear trend is that the surface energy decrease is more significant in the 2D semimetals (e.g. graphene, silicene, and germanene) than that in the 2D semiconductors (e.g. TMDCs). This reflects the fact that the effective mass of carriers in the $2 \mathrm{D}$ semiconductors is much higher than that in the $2 \mathrm{D}$ semimetals, ${ }^{43}$ thereby resulting in high DOS, as well as a high $C_{2 \mathrm{D}}$ (see eq 3). This concept also explains why the surface energy decrease for silicene and germanene are lower than that for graphene. ${ }^{47}$ To our knowledge, the doping-induced surface energy change in 2D materials has never been investigated experimentally, which may be of interest for future study. 


\section{Reorientation of Liquid Molecules}

Next, we discuss the interactions between 2D materials and liquid. In a doped 2D material, the delocalized carriers are confined in the 2D plane. Therefore, following the spirit of the mean-field theory, we treat it as a continuously, uniformly charged surface. Since these charges are either generated by interacting with the underlying substrate, or electrostatically induced by gating, the electroneutrality still holds before in contact with liquid. The surface charges result in two consequences that may change $\gamma_{2 \mathrm{D}-\mathrm{L}}$, including (i) the reorientation of adjacent liquid molecules ${ }^{36}$ and (ii) the formation of the electric double layer (EDL) at the liquid-solid interface, known as the electrowetting effect. ${ }^{48,49}$ Under the assumption that the vdW and electrostatic (Coulombic) interactions are additive, which compose the interfacial tension, we propose that the interfacial tension change is given by:

$$
\Delta \gamma_{2 \mathrm{D}-\mathrm{L}}=\Delta \gamma_{2 \mathrm{D}-\mathrm{L}}^{\text {Orien }}+\Delta \gamma_{2 \mathrm{D}-\mathrm{L}}^{\mathrm{EDL}}
$$

where $\Delta \gamma_{2 \mathrm{D}-\mathrm{L}}^{\text {Orien }}$ and $\Delta \gamma_{2 \mathrm{D}-\mathrm{L}}^{\mathrm{EDL}}$ correspond to the contributions from the reorientation and the EDL effects, respectively. We also assume that $\Delta \gamma_{2 \mathrm{D}-\mathrm{L}}^{\text {Orien }}$ is independent of the electrolyte concentration, since the concentration of liquid molecules is typically orders-of-magnitude higher.

First we discuss the orientation effect. Understanding the reorientation effect involves positioning and sampling the collective, time-averaged motion of liquid molecules near the interface, which is a standard molecular dynamics (MD) problem. Note that even with the state-of-the-art MD algorithms, it remains challenging to accommodate the calculations for the EDL, in which the length scale of electric field can be larger than one micrometer in diluted electrolyte solutions, e.g., pure water with self-ionized $\mathrm{H}_{3} \mathrm{O}^{+}$and $\mathrm{OH}^{-}$ions. Here we consider the graphene-water interface as a model system. All MD simulations were

carried out using the GROMACS 4.5 software package. ${ }^{50}$ Monolayer graphene was modeled as an infinite rigid sheet in the $\mathrm{x}-\mathrm{y}$ plane. The carbon atoms of graphene were treated as 
uncharged Lennard-Jones (LJ) spheres with $\sigma=0.34 \mathrm{~nm}$ and $\epsilon=0.223 \mathrm{~kJ} / \mathrm{mol},{ }^{51}$ using the force-field parameters reported by Tummala and Striolo. ${ }^{52}$ The doping effect is included by assigning an equal amount of charge $\sigma_{2 \mathrm{D}} / \rho_{\mathrm{G}}$, where $\rho_{\mathrm{G}}$ is the surface density of carbon atoms, to each carbon atom. The $\sigma_{2 \mathrm{D}}$ range considered here approximately correspond to the partial atomic charge from -0.012 to $0.012 \mathrm{e} /$ atom. Water molecules were modeled using the SPC/E model ${ }^{53}$ with bond lengths and angles of water molecules constrained using the SETTLE algorithm. ${ }^{54}$ Lennard-Jones interactions were treated with a cutoff distance of 1 $\mathrm{nm}$, with those between different atoms calculated using the standard geometric averaging rule. Long-range electrostatic interactions were treated using the particle mesh Ewald (PME) summation method ${ }^{55,56}$ with a short-range cutoff distance of $1 \mathrm{~nm}$. The velocity-rescaled Berendsen thermostat was implemented to maintain a constant system temperature of 298.15 K. ${ }^{57}$ All simulations were carried out under the NVT ensemble, and the equations of motion of water molecules were integrated over a range of $20 \mathrm{~ns}$ with $10^{7}$ steps.

In order to precisely determine the interfacial interactions using MD simulations, instead of the commonly used model that compared the nanoscale contact angle by placing a nanodroplet onto a sheet of suspended 2D material, ${ }^{36-39}$ we simulate the difference of the total potential energy, E, between two separate systems that contain (i) only water molecules with two surfaces exposing to vacuum (L), and (ii) the same amount of water molecules with one surface in contact with graphene (placed at $z=0$ ) and the other surface exposing to vacuum (GL) (see Figure 3(a) and 3(b)). Periodic boundary conditions are used in all three directions of the simulation boxes in both systems. Additionally, a vacuum layer of 3 $\mathrm{nm}$ thick along the $z$-axis is placed to separate the periodic images of the graphene-water system. The total energy in both systems can be formulated as: $E_{\mathrm{L}}=\mu_{\mathrm{L}} n_{\mathrm{L}}+2 \gamma_{\mathrm{L}} S$ and $E_{\mathrm{GL}}=\mu_{\mathrm{L}} n_{\mathrm{L}}+\left(\gamma_{\mathrm{L}}+\gamma_{2 \mathrm{D}-\mathrm{L}}+\gamma_{2 \mathrm{D}}\right) S$, respectively, where $\mu_{\mathrm{L}}$ is the chemical potential per water molecule in the bulk phase, $n_{\mathrm{L}}$ is the number of liquid molecules in the simulation box, and $S$ is the area of the $x y$-plane. A simulation box with a sufficiently large length of $21 \mathrm{~nm}$ in the $z$-direction is used, with a 18 -nm thick block of water molecules, to minimize the effect of the 
long-range electrostatic interaction between the charged graphene and the water molecules at the water-vacuum interface, by ensuring that the time-averaged dipole moment for the water molecules at the water-vacuum interface approaches zero (see Supporting Information Figure S1). One can show that $E_{\mathrm{GL}}-E_{\mathrm{L}}=\left(\gamma_{2 \mathrm{D}-\mathrm{L}}+\gamma_{2 \mathrm{D}}-\gamma_{L}\right) S=\left(\Phi+2 \gamma_{2 \mathrm{D}}\right) S$, where $\Phi$ is the interfacial energy, which is defined as $\Phi=\gamma_{2 \mathrm{D}-\mathrm{L}}-\gamma_{2 \mathrm{D}}-\gamma_{\mathrm{L}}$, and combining with eq 1 , the change of interfacial energy $\Delta \Phi$ can be formulated as:

$$
\Delta \Phi=\Delta\left(E_{\mathrm{GL}}-E_{\mathrm{L}}\right) / S-2 \Delta \gamma_{2 \mathrm{D}}=-\gamma_{\mathrm{L}}(\Delta \cos \theta)^{\text {Orien }}
$$

where $(\Delta \cos \theta)^{\text {Orien }}$ corresponds to the contact angle change due to the reorientation effect. Note that in the current MD simulation setup, the surface tension of the 2D material remains unchanged (i.e. $\Delta \gamma_{2 \mathrm{D}}=0$ ), since the Fermi level change with respect to $\sigma_{2 \mathrm{D}}$ (see eq 3 ) is not included in the algorithm. In all simulations we assume a constant surface tension of water $\gamma_{\mathrm{L}}=72.8 \mathrm{~mJ} \cdot \mathrm{m}^{-2}$. Accordingly, $\Delta \Phi$ follows $\Delta \Phi=\Delta\left(E_{\mathrm{GL}}-E_{\mathrm{L}}\right) / S=\Delta \Phi_{\mathrm{LJ}}+\Delta \Phi_{\text {Coul }}$, where $\Delta \Phi_{\mathrm{LJ}}$ and $\Delta \Phi_{\text {Coul }}$ correspond to the change for the $\mathrm{vdW}$ and Coulombic interaction potentials, respectively.

Figure 3(c) shows the values of $\Delta \Phi$, and its contributions from the Lennard-Jones $\left(\Delta \Phi_{\mathrm{LJ}}\right)$ and Coulombic interactions $\left(\Delta \Phi_{\text {Coul }}\right)$, as functions of $\sigma_{2 \mathrm{D}}$. We note that the change of adhesion energy in the doped graphene system is dominated by the Coulombic interaction. When the doping level of graphene is $\pm 4 \times 10^{13} \mathrm{e} \cdot \mathrm{cm}^{-2}$, the Coulombic interaction causes a decrease in the adhesion energy of $\sim-15 \mathrm{~mJ} \cdot \mathrm{m}^{-2}$, while the $\mathrm{vdW}$ interaction, on the other hand, causes a slight increase in the adhesion energy by less than $5 \mathrm{~mJ} \cdot \mathrm{m}^{-2}$. In other words, concerning the reorientation of water molecules at large doping levels, the Coulombic interaction favors the decrease of $\gamma_{2 \mathrm{D}-\mathrm{L}}$, and thus the contact angle $\theta$, while the vdW interaction slightly increases $\gamma_{2 \mathrm{D}-\mathrm{L}}$ and $\theta$. We shall note that due to the highly polar nature of water molecules, the average inter-molecular equilibrium distance in the absence of external electric field $\left(r_{\mathrm{O}-\mathrm{O}}=2.75 \AA\right)$ is shorter than the Lennard-Jones equilibrium distance 
$\left(r_{\mathrm{O}-\mathrm{O}}=3.16 \AA\right) .{ }^{58}$ Increasing the doping density of the graphene sheet essentially enhances the Coulombic attractions between graphene and water molecules, further reducing the intermolecular equilibrium distance, as seen in Figure S2(a), the distance between the first water layer and graphene (calculated from maximal $\rho_{\mathrm{L}}$ from Figure $3(\mathrm{~d})$ ) slightly decreases in doped graphene systems, compared with the charge-neutral system, which eventually leads to a slight increase in $\Delta \Phi_{\mathrm{LJ}}$. The plot of $\Delta \Phi$ as a function of $\sigma_{2 \mathrm{D}}$ in Figure $3(\mathrm{c})$ shows an apparent asymmetric behavior. This can be further revealed from the $z$-dependent local molecular density $\rho_{\mathrm{L}}$ and charge density $\delta_{\mathrm{L}}$ profiles of the water molecules, as shown in Figure 3(d) and 3(e), respectively. The molecular density of the first water layer adjacent to graphene increases when graphene is p-doped $\left(\sigma_{2 \mathrm{D}}=0.012 e \cdot \mathrm{cm}^{-2}\right.$, or equivalently 4.56 $e /$ atom $)$ and decreases when graphene is n-doped $\left(\sigma_{2 \mathrm{D}}=-0.012 e \cdot \mathrm{cm}^{-2}\right.$, or equivalently -4.56 e/atom) compared with the case of charge-neutral graphene, indicating the polaritydependent adsorption of water molecules on graphene: lighter $\mathrm{H}$ atoms prefer to adsorb onto n-doped graphene electrostatically. Similar trend can also be observed in the $\delta_{\mathrm{L}}$ (Figure 3(e))and water dipole orientation $\cos \mu$ (Figure S1) profiles, both suggesting that water orientation depends strongly on $\sigma_{2 \mathrm{D}}$ : more negative $\sigma_{2 \mathrm{D}}$ tends to orient the water dipoles more towards the 2D interface, reflected by the more positive charge densities due to firstlayer $\mathrm{H}$ atoms, and the more negative $\cos \mu$ values. Interestingly, the $\Delta \Phi_{\text {Coul }}$ exhibits small positive contribution for slightly n-doped graphene, when $\sigma_{2 \mathrm{D}} \approx-2 \times 10^{13} e \cdot \mathrm{cm}^{-2}$ or equivalently $-0.76 \mathrm{e} /$ atom, indicating that the reorientation of interfacial water molecules by small negative charges on graphene helps minimizing the Coulombic interactions. The trend observed in hydrogen-bond densities (see Figure S3) also matches those observed in $\rho_{\mathrm{L}}, \delta_{\mathrm{L}}$ and $\cos \mu$ profiles.

Finally, we believe that the asymmetric behavior of $\Delta \Phi_{\text {Coul }}$ with respect to $\sigma_{2 \mathrm{D}}$ is due to the nonlinear decrease in $\delta_{\mathrm{L}}$ of the first water layer, when graphene becomes more n-doped (see Figure $\mathrm{S} 2(\mathrm{~d})$ ). As a general trend, $\delta_{\mathrm{L}}$ of the first water layer increases when $\sigma_{2 \mathrm{D}}$ becomes more negative, due to the attraction of $\mathrm{H}$ atoms to the graphene surface. However we observe 
a decrease of $\delta_{\mathrm{L}}$ when $\sigma_{2 \mathrm{D}}$ ranges from -0.001 to $-0.006 e \cdot \mathrm{cm}^{-2}$ (see Figure $\mathrm{S} 2(\mathrm{~d})$ ), which could be a combined result of the graphene-water Coulombic interactions and intermolecular hydrogen bonding. We also notice a nonlinear behavior in the $\rho_{\mathrm{L}}$ of the first water layer: when $\sigma_{2 \mathrm{D}}$ becomes more negative, the interatomic distance $r_{\mathrm{C}-\mathrm{O}}$ between graphene $\mathrm{C}$ and water $\mathrm{O}$ atoms will increase due to Coulombic repulsion. Note that the increased Coulombic repulsion energy for $\mathrm{C}-\mathrm{O}$ pairs from more negative $\sigma_{2 \mathrm{D}}$ values is balanced by the increased vdW attractions between $\mathrm{C}-\mathrm{O}$ pairs when $r_{\mathrm{C}-\mathrm{O}}$ increases, which eventually prevents the reduction of the first water layer density at an equilibrium C-O distance, as seen at $\sigma_{2 \mathrm{D}}<-0.01 e \cdot \mathrm{cm}^{-2}$ (Figure S2(b)). Here we propose a simple capacitor-like model, considering the contributions from $\delta_{\mathrm{L}}$ of the first water layer of the first water layer and $\sigma_{2 \mathrm{D}}$ to the interfacial Coulombic energy $\Phi_{\text {Coul }}^{\mathrm{int}}$ (see the discussion in Supporting Information and Figure S4). We show that in the n-doped graphene system the charge density of the first water layer has a major contribution to $\Delta \Phi_{\text {Coul }}$. The nonlinear behavior of $\delta_{\mathrm{L}}$ at low n-doping level is responsible for the shift of maximum of the $\Delta \Phi_{\text {Coul }}-\sigma_{2 \mathrm{D}}$ curve. The analysis further indicates the importance of interfacial water configuration in determining the change of the interaction potentials.

Although the MD simulation results show that the Coulombic interactions between the doped graphene and water molecules dominate the reorientation effect, such conclusion may not be readily applied to other types of $2 \mathrm{D}$ materials, as the intensity of vdW interactions and surface charge polarization can vary greatly. First, the microscopic vdW and Coulombic interactions on 2D materials depend on the lattice constant and atomic density. In addition, for homoatomic 2D materials such as graphene, silicene, germanene and phosphorene, their surface charge polarization is negligible compared to heteroatomic 2D materials, for instance TMDC. It has been shown that, for TMDCs, the value of $\gamma_{2 \mathrm{D}-\mathrm{L}}$ is already largely determined by the Coulombic interactions in the charge-neutral systems. ${ }^{17,21}$ Therefore we anticipate that case-by-case studies and DFT calculations which deal with the post-doping charge redistribution, might be required, as shown by several previous studies. ${ }^{17,59,60}$ 


\section{Electrical Double Layer Effect}

The EDL effect that decreases the interfacial tension has been extensively studied in the context of the electrowetting phenomena, ${ }^{61}$ in which the interfacial tension is reduced due to the adsorption of ionic species at the solid-liquid interface. Here we extend the concept to the 2D material-liquid systems. Consider a closed system containing an interface formed between liquid and a sheet of free-standing 2D material, by combining with the Gibbs adsorption theory, ${ }^{42}$ one can obtain:

$$
\mathrm{d} \gamma_{2 \mathrm{D}-\mathrm{L}}^{\mathrm{EDL}}=-\sigma_{2 \mathrm{D}} \mathrm{d} \psi_{2 \mathrm{D}}-\sum_{\mathrm{i}} \Gamma_{\mathrm{i}} \mathrm{d} \mu_{\mathrm{i}}
$$

where $\Gamma_{\mathrm{i}}$ and $\mu_{\mathrm{i}}$ are the interfacial excess and the chemical potential of the ionic specie $i$ at the interface, respectively. Relative to that in the bulk phase $(\psi=0)$, the interfacial chemical potential of a charged solute $\mu_{\mathrm{i}}$, is given by $\mu_{\mathrm{i}}=z_{\mathrm{i}} e \psi_{2 \mathrm{D}}$, where $z_{\mathrm{i}}$ is the valency of component $i$, under the assumption that the interfacial electric potential is equal to $\psi_{2 \mathrm{D}}$. As with the approach leading to eq 3 , it follows:

$$
\Delta \gamma_{2 \mathrm{DDL}-\mathrm{L}}^{\mathrm{EDL}}=-\int_{0}^{\sigma_{2 \mathrm{D}}} \sigma^{\prime}\left(\frac{1}{C_{2 \mathrm{D}}}\right) \mathrm{d} \sigma^{\prime}-\int_{0}^{\sigma_{\mathrm{L}}} \sigma^{\prime}\left(\frac{1}{C_{\mathrm{EDL}}}\right) \mathrm{d} \sigma^{\prime}
$$

where $\sigma_{\mathrm{L}}$ is the interfacial charge density per unit area in the liquid phase, and $C_{\mathrm{EDL}}$ is the EDL capacitance. Combining eq 7 with eqs 1 and 3, the contact angle change due to the EDL effect, $(\Delta \cos \theta)^{\mathrm{EDL}}$ is given by:

$$
(\Delta \cos \theta)^{\mathrm{EDL}}=-\frac{1}{\gamma_{\mathrm{L}}} \int_{0}^{\sigma_{\mathrm{L}}} \sigma^{\prime}\left(\frac{1}{C_{\mathrm{EDL}}}\right) \mathrm{d} \sigma^{\prime}
$$

In other words, eqs 3 and 7 suggest that $\sigma_{2 \mathrm{D}}$ decreases both $\gamma_{2 \mathrm{D}}$ and $\gamma_{2 \mathrm{D}-\mathrm{L}}$ to the same

extent, i.e. $\int_{0}^{\sigma_{2 \mathrm{D}}} \sigma^{\prime} / C_{2 \mathrm{D}} \mathrm{d} \sigma^{\prime}$, such that the contribution of quantum capacitance cancels out in the YLE.

For a $z$ : $z$ electrolyte solution, with the molar concentration $c_{0}$ and the electrolyte 
valence $z$, we describe the electric potential profile in liquid with the one-dimensional GouyChapman-Stern model, ${ }^{62}$ as schematically shown in Figure 4(a). The EDL consists of a Helmholtz layer with the permittivity of liquid $\epsilon_{\mathrm{L}}$ and thickness $d_{\mathrm{H}}$, next to the GouyChapman (GC) diffuse layer where the electric potential profile follows the Poisson Boltzmann equation. ${ }^{42}$ The $C_{\mathrm{EDL}}$ of the Gouy-Chapman-Stern model is equivalent to two capacitors in series, namely,

$$
\frac{1}{C_{\mathrm{EDL}}}=\frac{1}{C_{\mathrm{H}}}+\frac{1}{C_{\mathrm{GC}}}
$$

where $C_{\mathrm{H}}=\epsilon_{\mathrm{L}} / d_{\mathrm{H}}$ is the capacitance of the Helmholtz double layer and $C_{\mathrm{GC}}=\sqrt{\frac{2 z^{2} e^{2} \epsilon_{\mathrm{L}} c_{0} N_{\mathrm{A}}}{k_{\mathrm{B}} T}} \cosh \left(\frac{z e \psi_{\mathrm{GC}}}{2 k_{\mathrm{B}} T}\right)$ is the differential capacitance of the GC layer, with $\psi_{\mathrm{GC}}$ corresponding to the electric potential at the GC interface. Consider a Gaussian enclosure including the 2D material layer and the EDL, due to a zero net electric field at the surface of the Gaussian enclosure, the electroneutrality of the system ${ }^{42}$ suggests:

$$
\sigma_{2 \mathrm{D}}+\sigma_{\mathrm{L}}=0
$$

And therefore, the electric potentials corresponding to the surface of the 2D material and the outer Helmholtz plane, $\psi_{2 \mathrm{D}}$ and $\psi_{\mathrm{GC}}$, are given by:

$$
\begin{aligned}
& \psi_{\mathrm{GC}}=-\frac{2 k_{\mathrm{B}} T}{z e} \sinh ^{-1}\left(\frac{-\sigma_{2 \mathrm{D}}}{\sqrt{8 c_{0} N_{\mathrm{A}} \epsilon_{\mathrm{L}} k_{\mathrm{B}} T}}\right) \\
& \psi_{2 \mathrm{D}}=\psi_{\mathrm{GC}}+\sigma_{2 \mathrm{D}} \frac{d_{\mathrm{H}}}{\epsilon_{\mathrm{L}}}
\end{aligned}
$$

where $N_{\mathrm{A}}$ is the Avogadro constant and $k_{\mathrm{B}}$ is the Boltzmann constant. Accordingly, the final form of $(\Delta \cos \theta)^{\mathrm{EDL}}$ is given by:

$$
\begin{aligned}
(\Delta \cos \theta)^{\mathrm{EDL}} & =\frac{\sigma_{2 \mathrm{D}}^{2}}{2 \gamma_{\mathrm{L}} C_{\mathrm{H}}} \frac{1}{\gamma_{\mathrm{L}}} \sqrt{\frac{32 k_{\mathrm{B}}^{3} T^{3} \epsilon_{\mathrm{L}} c_{0} N_{\mathrm{A}}}{z^{2} e^{2}}}\left[\cosh \left(\frac{z e \psi_{\mathrm{GC}}}{2 k_{\mathrm{B}} T}\right)-1\right] \\
& =\frac{\Delta \sigma_{2 \mathrm{D}}^{2}}{2 \gamma_{\mathrm{L}}}\left[\frac{1}{C_{\mathrm{H}}}+\frac{1}{\left(C_{\mathrm{DH}}+C_{\mathrm{GC}}\right) / 2}\right]
\end{aligned}
$$


where $C_{\mathrm{DH}}=\epsilon_{\mathrm{L}} / \lambda_{\mathrm{D}}$ is the differential capacitance of the diffuse layer using the Debye-Hückel approximation, ${ }^{42}$ and $\lambda_{\mathrm{D}}=\sqrt{\frac{\epsilon_{\mathrm{L}} k_{\mathrm{B}} T}{2 z^{2} e^{2} c_{0} N_{\mathrm{A}}}}$ is the Debye screening length. Compared with the classical YLE, $\Delta \cos \theta=-\frac{\sigma^{2}}{2 \gamma_{\mathrm{L}} C_{\mathrm{YL}}}$, where $C_{\mathrm{YL}}$ is the equivalent capacitance in YLE, one can show that $C_{\mathrm{YL}}$ is given by:

$$
\frac{1}{C_{\mathrm{YL}}}=\frac{1}{C_{\mathrm{H}}}+\frac{1}{\left(C_{\mathrm{DH}}+C_{\mathrm{GC}}\right) / 2}
$$

The above analysis leads to two important observations: (i) If a thin contamination layer (usually composed of charge-neutral hydrocarbon species ${ }^{18}$ ) exists between the Helmholtz layer and the 2D material surface, the electroneutrality at the interface (eq 10) still holds, such that $(\Delta \cos \theta)^{\mathrm{EDL}}$ remains the same (see eq 12). In other words, despite the recent debate about the role of the airborne adsorbates in the contact angle measurement on 2D materials, ${ }^{15,18,19,21}$ we expect that the experimental characterization of the doping-induced contact angle change is more robust and reproducible. (ii) As shown in eqs 9 and 7 , the equivalent capacitance in the YLE, $C_{\mathrm{YL}}$, is not identical to the total capacitance in the EDL, $C_{\mathrm{EDL}}$. We notice that the literature in this field (e.g. Refs. 34-36,39) often simply assumed that $C_{\mathrm{YL}}=C_{\mathrm{DH}}$ in the YLE, which significantly overestimates $(\Delta \cos \theta)^{\mathrm{EDL}}$, when $\psi_{2 \mathrm{D}} \gg k_{\mathrm{B}} T / e,{ }^{63}$ as can be found in heavily-doped $2 \mathrm{D}$ materials.

Consider the interface formed between graphene and an (1:1) aqueous solution, using the parameters of $d_{\mathrm{H}}=3 \AA,{ }^{64}$ and $\gamma_{\mathrm{L}}=72.8 \mathrm{~mJ} \cdot \mathrm{m}^{-2}$ at $T=298 \mathrm{~K}$, the calculated $(\Delta \cos \theta)^{\mathrm{EDL}}$ as a function of $\sigma_{2 \mathrm{D}}$ is shown in Figure $4(\mathrm{~b})$. Note that $c_{0}=10^{-7} \mathrm{~mol} \cdot \mathrm{L}^{-1}$ corresponds to the pure water system. We find that the contact angle change is stronger in a more diluted electrolyte solution, resulting from an increase in Debye screening length $\lambda_{\mathrm{D}}$, which in turn decreases both $C_{\mathrm{GC}}$ and $C_{\mathrm{DH}}$. This is distinct from the that in the typical electrowetting experiment on a dielectric layer, in which $C_{\mathrm{YL}}$ is governed by the capacitance of the dielectric layer, so the contact angle change is almost independent of the electrolyte concentration. ${ }^{61}$ Moreover, within the range of $\sigma_{2 \mathrm{D}}$ considered here $\left(-4 \times 10^{13} \sim 4 \times 10^{13} e \cdot \mathrm{cm}^{-2}\right)$, the calculated 
$(\Delta \cos \theta)^{\mathrm{EDL}}$ is always less than 0.06. In combination with the $\Delta \gamma_{2 \mathrm{D}-\mathrm{L}}^{\text {Orien }}$ (equivalent to $\Delta \Phi$, see previous section) calculated by the MD simulations, the total change of contact angle $(\Delta \cos \theta)^{\text {Orien+EDL }}$ due to the doping effect is shown in Figure 4(c). We separately fit the $\Delta \Phi_{\mathrm{LJ}}$ and $\Delta \Phi_{\text {Coul }}$ from the reorientation effect as functions of $\sigma_{2 \mathrm{D}}$ in n- and p-doped regimes, respectively (see Figure S5), and use the fitting results to construct the $\Delta \Phi-\sigma_{2 \mathrm{D}}$ relation. We find that the reorientation effect is more predominant than the EDL effect, therefore the overall change of contact angle is less dependent on the solute concentration, compared with Figure 4(b).

Together with the reorientation effect, the calculated $\Delta \cos \theta$ change only reaches $0.06 \sim$ 0.08, corresponding to a maximum contact angle decrease of $3.5 \sim 4.5^{\circ}$, for $\sigma_{2 \mathrm{D}}= \pm 1 \times 10^{13} e$. $\mathrm{cm}^{-2}$, which is is a typical range for both the electrostatic gating ${ }^{33}$ and substrate-induced doping. ${ }^{35}$ Clearly, the predicted contact angle change upon doping is still lower than those observed experimentally. In the next section, we discuss the practical considerations that may influence a 2D material-coated surface.

\section{Practical Considerations}

In practice, in order to carry out the contact angle measurement, a large-area sheet of $2 \mathrm{D}$ material is required to cover the surface. Therefore, most of the experimental findings are based on the chemical-vapor-deposited (CVD) samples, which are inherently polycrystalline with grain boundaries and point defects. ${ }^{65}$ In addition, the transfer of the ultrathin films onto a solid surface often results in nanometer- to micrometer- scale tears and wrinkles. Although our analysis has suggested that the charge-neutral polymer residues and airborne contaminations do not influence $(\Delta \theta)^{\mathrm{EDL}}$, here we show that the defect density plays an important role in determining the wettability of doped 2D materials.

Figures 5(a) and 5(b) illustrate how an electric field, either from the dopants on the substrate surface or from the electrostatic gating, penetrate through a void in a 2D material sheet and interact with the liquid phase directly. As a result, the EDL is built up adjacent 
to the substrate surface, with the surface excess $\sigma_{\mathrm{v}}$ and the effective capacitance $C_{\mathrm{v}}$. Since $\sigma_{v}$ is equivalent to $\sigma_{2 \mathrm{D}}$, together with the EDL and the reorientation effects discussed earlier, the modified YLE considering a 2D material with voids follows:

$$
\Delta \cos \theta=-f \frac{\sigma_{2 \mathrm{D}}^{2}}{\gamma_{\mathrm{L}} C_{\mathrm{v}}}+(1-f)\left[(\Delta \cos \theta)^{\text {Orien }}+(\Delta \cos \theta)^{\mathrm{EDL}}\right]
$$

where $f$ is the void (defect) fraction in the 2D material. For the electrostatic gating experiments (see Figure 5(a)), the 2D material quantum capacitor and the dielectric capacitor are connected in series, so that the voltage applied between the gate electrode and 2D material, $V_{\mathrm{G}}$, is given by: ${ }^{45}$

$$
V_{\mathrm{G}}=\frac{\sigma_{2 \mathrm{D}}-\sigma_{0}}{C_{\mathrm{d}}}+\int_{\sigma_{0}}^{\sigma_{2 \mathrm{D}}} \frac{1}{C_{2 \mathrm{D}}} \mathrm{d} \sigma^{\prime}
$$

where $C_{\mathrm{d}}$ is the capacitance of the dielectric layer, and $\sigma_{0}$ is the initial doping density of the 2D material, corresponding to $V_{\mathrm{G}}=0$.

Next, in order to examine the effect of incomplete 2D material coverage, two independent sets of experimental results, which measure the water contact angle on (i) substrate-doped graphene $^{35}$ and (ii) electrostatically-gated graphene ${ }^{33}$ are chosen to compare, with $C_{\mathrm{v}}=C_{\mathrm{DH}}$ and $C_{\mathrm{v}}=C_{\mathrm{d}}$ in eq 14 , respectively. The parameter $f$ and $\sigma_{0}$ are determined by leastsquare fitting the experimentally-observed $\Delta \cos \theta$ with respect to $\sigma_{2 \mathrm{D}}$ using eq 14 . Figure 5 (c) compares the calculated $\Delta \cos \theta$ as a function of $\sigma_{2 \mathrm{D}}$, together with the two sets of experimental data. In both cases considered, we observe a slight degree of shift in the minima of the fitted curves, corresponding to the CNP of graphene, or $\sigma_{2 \mathrm{D}}=0$. In other words, we observe $\sigma_{0} \neq 0$ in both cases, which is typical for CVD-grown graphene samples. ${ }^{34,66}$ The fitted values of $f$ are reasonably small (3.6\% for the electrostatically-gated graphene and $3.0 \%$ for substrate-doped graphene), clearly demonstrating that the contact angle change can be greatly influenced by the defect density. We believe this explains the discrepancy between the experimental observations and the multiscale theoretical framework proposed here. 
Finally, we discuss the influence of 2D material choice under electrostatic gating condition. The above analysis (eq 12) has clearly suggested that the contact angle change effect $(\Delta \cos \theta)^{\mathrm{EDL}}$ only depends on $\sigma_{2 \mathrm{D}}$, which can be controlled by an electric displacement field, with the experimental setup shown in Figure 6(a). Specifically, eqs 11 and 15 suggest:

$$
\begin{aligned}
\mathrm{d} V_{\mathrm{G}} & =\left(\frac{1}{C_{\mathrm{d}}}+\frac{1}{C_{2 \mathrm{D}}}\right) \mathrm{d} \sigma_{2 \mathrm{D}} \\
\mathrm{d} \psi_{2 \mathrm{D}} & =-\left(\frac{1}{C_{\mathrm{H}}}+\frac{1}{C_{\mathrm{GC}}}\right) \mathrm{d} \sigma_{\mathrm{L}}
\end{aligned}
$$

And since $\sigma_{2 \mathrm{D}}=-\sigma_{\mathrm{L}}$, the first derivative of $\psi_{2 \mathrm{D}}$ with respect to $V_{\mathrm{G}}$, namely $\beta$, is given by:

$$
\beta=\frac{\mathrm{d} \psi_{2 \mathrm{D}}}{\mathrm{d} V_{\mathrm{G}}}=\frac{\frac{1}{C_{\mathrm{H}}}+\frac{1}{C_{\mathrm{GC}}}}{\frac{1}{C_{\mathrm{d}}}+\frac{1}{C_{2 \mathrm{D}}}}
$$

The index $\beta$ here quantifies the tunability of the contact angle change by $V_{\mathrm{G}}$. Accordingly, a high degree of $\beta$ can be attained by increasing both $C_{\mathrm{d}}$ and $C_{2 \mathrm{D}}$, thereby introducing the dependence on the choice of 2D material.

Here we demonstrate such phenomenon by considering an electrowetting setup comprised of a thin, high- $k$ dielectric layer $\left(2 \mathrm{~nm} \mathrm{HfO}_{2}\right.$ layer with the relative permittivity $\left.\epsilon_{\mathrm{d}}=24.0\right)$ underlying a layer of monolayer 2D material (Figure 6(a)). As addressed earlier, due to the fact that $(\Delta \cos \theta)^{\text {Orien }}$ of graphene-water system may not be readily applied to other $2 \mathrm{D}$ materials, we compare the calculated $(\Delta \cos \theta)^{\mathrm{EDL}}$ as a function of $V_{\mathrm{G}}$, by using the DFTcalculated $C_{2 \mathrm{D}}-\sigma_{2 \mathrm{D}}$ relations for a variety of $2 \mathrm{D}$ materials, as shown in $6(\mathrm{~b})$. Clearly, as a consequence of the high quantum capacitance, the wettability of the 2D semiconductors considered is more tunable compared to that for the $2 \mathrm{D}$ semimetals. In other words, in a $2 \mathrm{D}$ semiconductor, the value required to reach a certain is lowered. We predict that the contact angle change $\Delta \cos \theta$ for the $2 \mathrm{D}$ semiconductors (e.g. TMDCs) can reach up to $0.22 \sim 0.25$ within the range of $V_{\mathrm{G}}$ considered, corresponding to an interfacial tension change of $15 \sim 18$ $\mathrm{mJ} \cdot \mathrm{m}^{-2}$. The analysis presented here suggests that the manipulation of a liquid droplet on a layer of $2 \mathrm{D}$ material doped by an electric displacement field may be feasible. ${ }^{61,67}$ 
An interesting implication is that, together with the recent development in engineering $2 \mathrm{D}$ materials' wetting translucency, ${ }^{22-25}$ in principle, upon doping, a 2D material becomes less "transparent" (or more screening) to both van der Waals and Coulombic interactions exerted from the underlying substrate, due to enhanced liquid-2D material interactions. That is to say, the doping level can be another control variable to modulate the molecular packing and epitaxial behavior on a 2D material-coated surface, which may bring new technology opportunity for a variety of applications.

\section{Conclusion}

In conclusion, we present a multiscale theoretical framework concerning the wettability of doped 2D materials, by considering: (i) the change of 2D materials surface energy, (ii) the molecular reorientation of liquid molecules adjacent to the interface, and (iii) the electrical double layer formed in the liquid phase. Taking graphene as an example, we show that the Coulombic interaction dominates the change of liquid-2D material interfacial tension, at both atomistic and continuum length scales. The latter two effects were found to be the major mechanisms responsible for the water contact angle change at the $2 \mathrm{D}$ material interfaces upon doping.

The doping-induced reorientation of liquid molecules at the graphene-liquid interface is revealed by MD simulations, which also allow decoupling of vdW and Coulombic energy contributions to the interfacial tension in the absence of electrolytes in liquid. It is found that the interfacial energy change is dominated by the Coulombic interactions. Our results also reveal an asymmetric change of graphene-water interfacial energy upon doping, such that slightly n-doped graphene can reorient interfacial water molecules to minimize electrostatic attractions, and therefore, slightly increase the interfacial energy.

On the hand, the EDL effect is calculated by a continuum model. Our analysis suggests that the reorientation effect is more predominant than the EDL effect, On the graphene- 
water interface, we predict that the combined reorientation and EDL effects can induce a significant change of the interfacial tension $\Delta \gamma_{2 \mathrm{D}-\mathrm{L}}$, up to $-15 \sim-20 \mathrm{~mJ} \cdot \mathrm{m}^{-2}$, at the doping level of $\pm 4 \times 10^{13} \mathrm{e} \cdot \mathrm{cm}^{-2}$. By adding the fitting parameter concerning the defect density, our theoretical framework can nicely describe the experimentally observed doping-induced contact angle change. Finally, based on the DFT-calculated quantum capacitances (QCs) for a variety of $2 \mathrm{D}$ materials, we predict that the wettability of $2 \mathrm{D}$ semiconductors (e.g., TMDCs) is more tunable under an electric displacement field, compared with 2D semimetals (e.g. graphene) due to their high quantum capacitances. Our findings reveal a complete picture for the modulation of the molecular interactions between liquid and a 2D monolayer upon doping. The multiscale theoretical framework proposed here is expected to shed light on the surface science of 2D materials, as well as to provide a quantitative estimation for the wettability of the doped 2D materials. We hope that the development for the $2 \mathrm{D}$ materialsbased functional surfaces in liquid manipulation, energy harvesting, and molecular epitaxy will be facilitated by the fundamental principles and theoretical insights presented here.

\section{Acknowledgement}

T.T and C.-J. S. are grateful for the financial support from the ETH startup funding. S.L. would like to acknowledge the startup funding from the Energy and Materials Initiative at the Florida State University. S.L. and L.Z. would like to acknowledge funding from the National Natural Science Foundation of China (Grant No. 51106027).

\section{Supporting Information Available}

The following files are available free of charge. Additional results for the MD simulation and analysis of nonlinear behavior of interfacial Coulombic interactions in charged graphenewater system. 


\section{References}

(1) Novoselov, K. S.; Jiang, D.; Schedin, F.; Booth, T. J.; Khotkevich, V. V.; Morozov, S. V.; Geim, A. K. Proc. Natl. Acad. Sci. 2005, 102, 10451-10453.

(2) Mas-Ballesté, R.; Gómez-Navarro, C.; Gómez-Herrero, J.; Zamora, F. Nanoscale 2011, 3, 20-30.

(3) Novoselov, K. S.; Mishchenko, A.; Carvalho, A.; Castro Neto, A. H. Science 2016, 353, $\operatorname{aac} 9439$.

(4) Boott, C. E.; Nazemi, A.; Manners, I. Angew. Chem. Int. Ed. 2015, 54, 13876-13894.

(5) Li, X.; Colombo, L.; Ruoff, R. S. Adv. Mater. 2016, 28, 6247-6252.

(6) Prasai, D.; Tuberquia, J. C.; Harl, R. R.; Jennings, G. K.; Bolotin, K. I. ACS Nano 2012, 6, 1102-1108.

(7) Feng, J.; Graf, M.; Liu, K.; Ovchinnikov, D.; Dumcenco, D.; Heiranian, M.; Nandigana, V.; Aluru, N. R.; Kis, A.; Radenovic, A. Nature 2016, 536, 197-200.

(8) Rafiee, J.; Rafiee, M. A.; Yu, Z.-Z.; Koratkar, N. Adv. Mater. 2010, 22, 2151-2154.

(9) Yin, J.; Li, X.; Yu, J.; Zhang, Z.; Zhou, J.; Guo, W. Nat. Nanotechnol. 2014, 9, $378-383$.

(10) Surwade, S. P.; Smirnov, S. N.; Vlassiouk, I. V.; Unocic, R. R.; Veith, G. M.; Dai, S.; Mahurin, S. M. Nat. Nanotechnol. 2015, 10, 459-464.

(11) Hernández, S. C.; Bennett, C. J. C.; Junkermeier, C. E.; Tsoi, S. D.; Bezares, F. J.; Stine, R.; Robinson, J. T.; Lock, E. H.; Boris, D. R.; Pate, B. D.; et al., ACS Nano 2013, $7,4746-4755$.

(12) Shi, Y.; Zhou, W.; Lu, A.-Y.; Fang, W.; Lee, Y.-H.; Hsu, A. L.; Kim, S. M.; Kim, K. K.; Yang, H. Y.; Li, L.-J.; et al., Nano Lett. 2012, 12, 2784-2791. 
(13) Kim, Y.; Cruz, S. S.; Lee, K.; Alawode, B. O.; Choi, C.; Song, Y.; Johnson, J. M.; Heidelberger, C.; Kong, W.; Choi, S.; et al., Nature 2017, 544, 340-343.

(14) Taherian, F.; Marcon, V.; van der Vegt, N. F. A.; Leroy, F. Langmuir 2013, 29, $1457-$ 1465.

(15) Kozbial, A.; Gong, X.; Liu, H.; Li, L. Langmuir 2015, 31, 8429-8435.

(16) Parobek, D.; Liu, H. 2D Mater. 2015, 2, 032001.

(17) Govind Rajan, A.; Sresht, V.; Pádua, A. A. H.; Strano, M. S.; Blankschtein, D. ACS Nano 2016, 10, 9145-9155.

(18) Li, Z.; Wang, Y.; Kozbial, A.; Shenoy, G.; Zhou, F.; McGinley, R.; Ireland, P.; Morganstein, B.; Kunkel, A.; Surwade, S. P.; Li, L.; Liu, H. Nat. Mater. 2013, 12, 925-931.

(19) Xu, K.; Heath, J. R. Nat. Mater. 2013, 12, 872-873.

(20) Kozbial, A.; Li, Z.; Conaway, C.; McGinley, R.; Dhingra, S.; Vahdat, V.; Zhou, F.; D’Urso, B.; Liu, H.; Li, L. Langmuir 2014, 30, 8598-8606.

(21) Chow, P. K.; Singh, E.; Viana, B. C.; Gao, J.; Luo, J.; Li, J.; Lin, Z.; Elías, A. L.; Shi, Y.; Wang, Z.; et al., ACS Nano 2015, 9, 3023-3031.

(22) Raj, R.; Maroo, S. C.; Wang, E. N. Nano Lett. 2013, 13, 1509-1515.

(23) Rafiee, J.; Mi, X.; Gullapalli, H.; Thomas, A. V.; Yavari, F.; Shi, Y.; Ajayan, P. M.; Koratkar, N. A. Nat. Mater. 2012, 11, 217-222.

(24) Shih, C.-J.; Wang, Q. H.; Lin, S.; Park, K.-C.; Jin, Z.; Strano, M. S.; Blankschtein, D. Phys. Rev. Lett. 2012, 109, 176101.

(25) Shih, C.-J.; Strano, M. S.; Blankschtein, D. Nat. Mater. 2013, 12, 866-869. 
(26) Chen, W.; Santos, E. J. G.; Zhu, W.; Kaxiras, E.; Zhang, Z. Nano Lett. 2013, 13, $509-514$.

(27) Varchon, F.; Feng, R.; Hass, J.; Li, X.; Nguyen, B. N.; Naud, C.; Mallet, P.; Veuillen, J.Y.; Berger, C.; Conrad, E. H.; et al., Phys. Rev. Lett. 2007, 99, 126805.

(28) Giovannetti, G.; Khomyakov, P. A.; Brocks, G.; Karpan, V. M.; van den Brink, J.; Kelly, P. J. Phys. Rev. Lett. 2008, 101, 026803.

(29) Das, A.; Pisana, S.; Chakraborty, B.; Piscanec, S.; Saha, S. K.; Waghmare, U. V.; Novoselov, K. S.; Krishnamurthy, H. R.; Geim, A. K.; Ferrari, A. C.; et al., Nat. Nanotechnol. 2008, 3, 210-215.

(30) Perera, M. M.; Lin, M.-W.; Chuang, H.-J.; Chamlagain, B. P.; Wang, C.; Tan, X.; Cheng, M. M.-C.; Tománek, D.; Zhou, Z. ACS Nano 2013, 7, 4449-4458.

(31) Muruganathan, M.; Sun, J.; Imamura, T.; Mizuta, H. Nano Lett. 2015, 15, 8176-8180.

(32) Huttmann, F.; Martínez-Galera, A. J.; Caciuc, V.; Atodiresei, N.; Schumacher, S.; Standop, S.; Hamada, I.; Wehling, T. O.; Blügel, S.; Michely, T. Phys. Rev. Lett. 2015, $115,236101$.

(33) Hong, G.; Han, Y.; Schutzius, T. M.; Wang, Y.; Pan, Y.; Hu, M.; Jie, J.; Sharma, C. S.; Müller, U.; Poulikakos, D. Nano Lett. 2016, 4447-4453.

(34) Goniszewski, S.; Adabi, M.; Shaforost, O.; Hanham, S. M.; Hao, L.; Klein, N. Sci. Rep. 2016, 6, 22858.

(35) Ashraf, A.; Wu, Y.; Wang, M. C.; Yong, K.; Sun, T.; Jing, Y.; Haasch, R. T.; Aluru, N. R.; Nam, S. Nano Lett. 2016, 4708-4712.

(36) Ostrowski, J. H. J.; Eaves, J. D. J. Phys. Chem. B 2014, 118, 530-536. 
(37) Ren, H.; Zhang, L.; Li, X.; Li, Y.; Wu, W.; Li, H. Phys. Chem. Chem. Phys. 2015, 17, 23460-23467.

(38) Taherian, F.; Leroy, F.; van der Vegt, N. F. A. Langmuir 2015, 31, 4686-4695.

(39) Daub, C. D.; Bratko, D.; Leung, K.; Luzar, A. J. Phys. Chem. C 2007, 111, 505-509.

(40) Lippmann, G. Ann. Chem. Phys. 1875, 5, 494-549.

(41) Shen, Y. R.; Ostroverkhov, V. Chem. Rev. 2006, 106, 1140-1154.

(42) Bard, A. J.; Faulkner, L. R.; Leddy, J.; Zoski, C. G. Electrochemical methods: fundamentals and applications; Wiley New York, 1980; Vol. 2.

(43) Davies, J. H. The Physics of Low-Dimensional Semiconductors; Cambridge University Press, 1997.

(44) Das Sarma, S.; Adam, S.; Hwang, E. H.; Rossi, E. Rev. Mod. Phys 2011, 83, 407-470.

(45) Tian, T.; Rice, P.; Santos, E. J. G.; Shih, C.-J. Nano Lett. 2016, 16, 5044-5052.

(46) John, D. L.; Castro, L. C.; Pulfrey, D. L. J. Appl. Phys. 2004, 96, 5180-5184.

(47) Yan, J.-A.; Stein, R.; Schaefer, D. M.; Wang, X.-Q.; Chou, M. Y. Phys. Rev. B 2013, $88,121403$.

(48) Lippmann, G. Journal de Physique Théorique et Appliquée 1908, 7, 821-825.

(49) Mugele, F.; Baret, J.-C. J. Phys.: Condens. Matter 2005, 17, R705-R774.

(50) Hess, B.; Kutzner, C.; van der Spoel, D.; Lindahl, E. J. Chem. Theory Comput. 2008, $4,435-447$.

(51) Cheng, A.; Steele, W. A. J. Chem. Phys. 1990, 92, 3858-3866.

(52) Tummala, N. R.; Striolo, A. J. Phys. Chem. B 2008, 112, 1987-2000. 
(53) Berendsen, H. J. C.; Grigera, J. R.; Straatsma, T. P. J. Phys. Chem. 1987, 91, 62696271.

(54) Miyamoto, S.; Kollman, P. A. J. Comput. Chem. 1992, 13, 952-962.

(55) Darden, T.; York, D.; Pedersen, L. J. Chem. Phys. 1993, 98, 10089-10092.

(56) Essmann, U.; Perera, L.; Berkowitz, M. L.; Darden, T.; Lee, H.; Pedersen, L. G. J. Chem. Phys. 1995, 103, 8577-8593.

(57) Bussi, G.; Donadio, D.; Parrinello, M. J. Chem. Phys. 2007, 126, 014101.

(58) Mark, P.; Nilsson, L. J. Phys. Chem. A 2001, 105, 9954-9960.

(59) Sresht, V.; Pádua, A. A. H.; Blankschtein, D. ACS Nano 2015, 9, 8255-8268.

(60) Sresht, V.; Govind Rajan, A.; Bordes, E.; Strano, M. S.; Pádua, A. A.; Blankschtein, D. J. Phys. Chem. C 2017, 121, 9022-9031.

(61) Mugele, F.; Baret, J.-C. J. Phys.: Cond. Mat. 2005, 17, R705-R774.

(62) Stern, O. Z. Elektrochem 1924, 30, 1014-1020.

(63) Israelachvili, J. N. Intermolecular and surface forces; Academic Press, 2003.

(64) Mcclendon, J. F. Science 1927, 66, 200-200.

(65) Banhart, F.; Kotakoski, J.; Krasheninnikov, A. V. ACS Nano 2011, 5, 26-41.

(66) Shih, C.-J.; Pfattner, R.; Chiu, Y.-C.; Liu, N.; Lei, T.; Kong, D.; Kim, Y.; Chou, H.-H.; Bae, W.-G.; Bao, Z. Nano Lett. 2015, 15, 7587-7595.

(67) Hayes, R. A.; Feenstra, B. J. Nature 2003, 425, 383-385. 


\section{Figures}

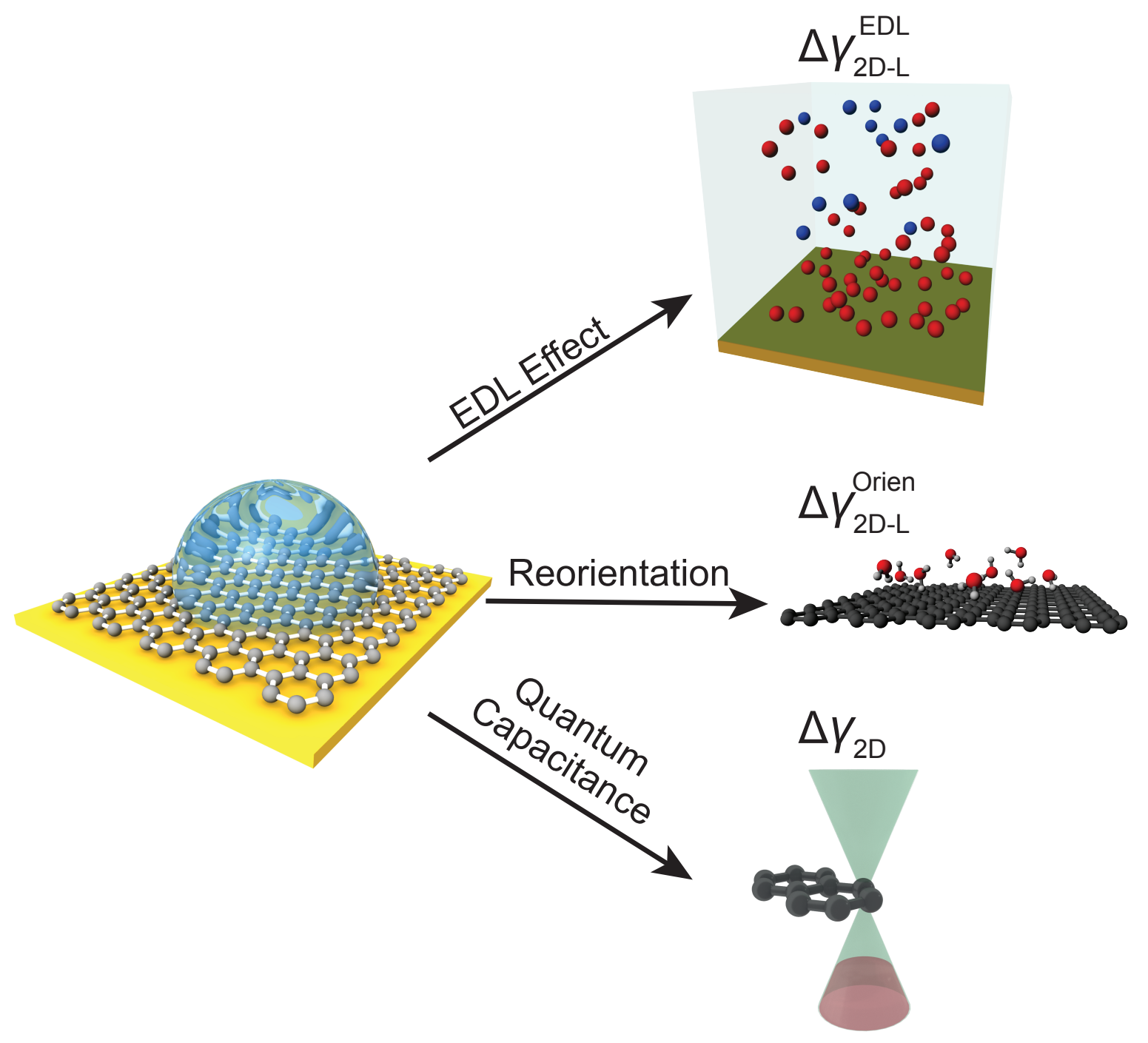

Figure 1: Scheme of the multiscale approach for modeling the doping-induced wettability tuning of $2 \mathrm{D}$ materials. 


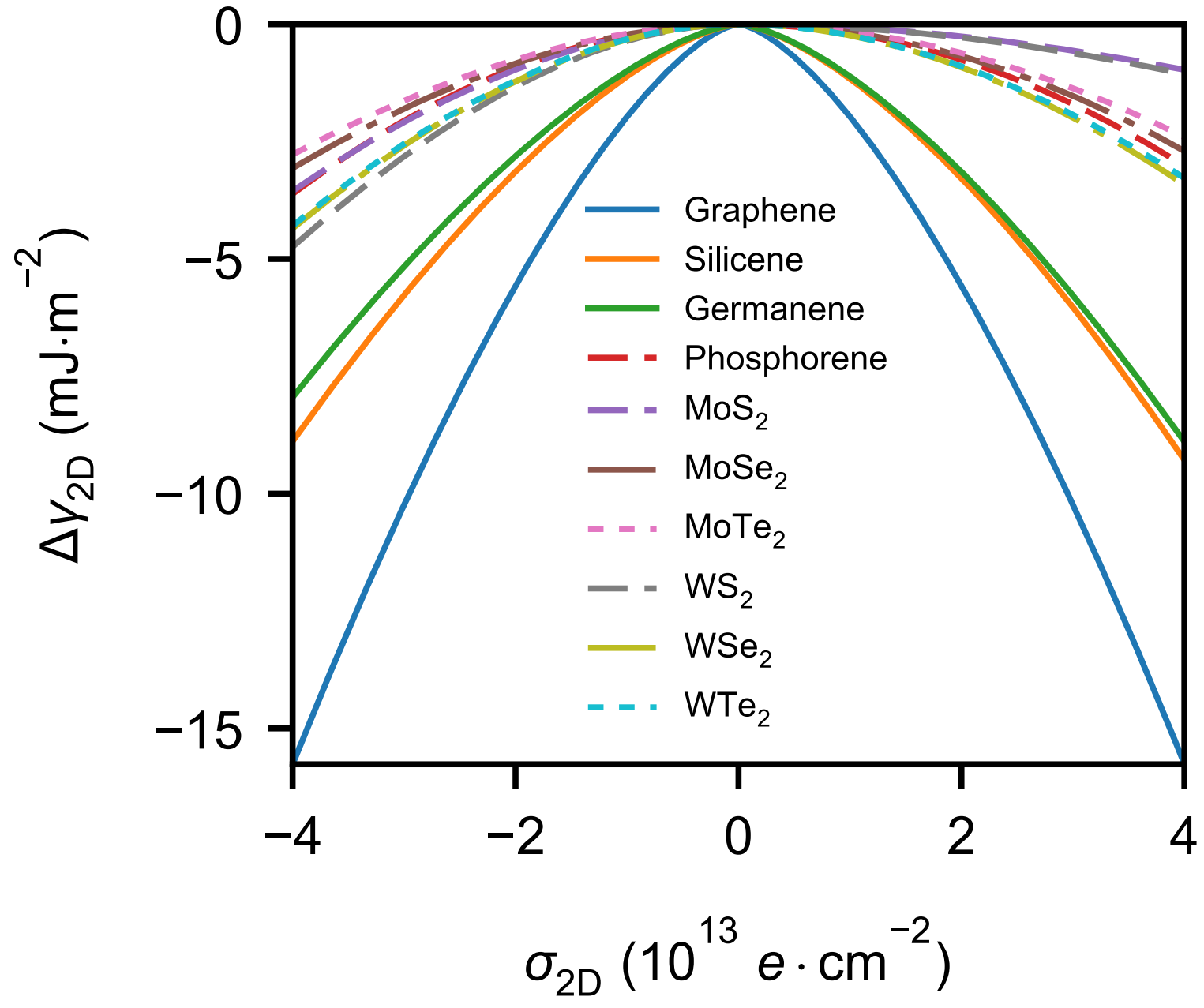

Figure 2: $\Delta \gamma_{2 \mathrm{D}}$ as a function of $\sigma_{2 \mathrm{D}}$ for selected 2D materials: graphene, silicene, germanene, phosphorene, $\mathrm{MoS}_{2}, \mathrm{MoSe}_{2}, \mathrm{MoTe}_{2}, \mathrm{WS}_{2}, \mathrm{WSe}_{2}$ and $\mathrm{WTe}_{2}$ 
(a)

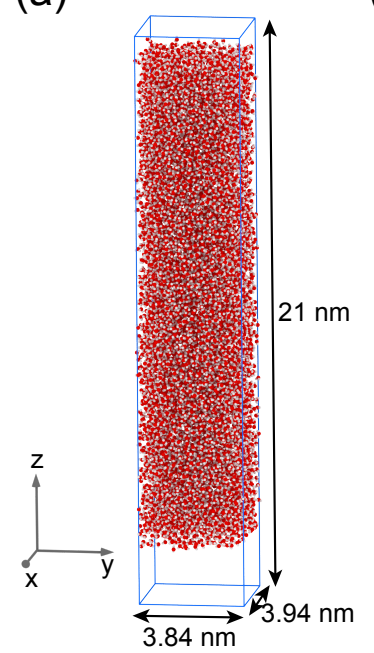

(d)

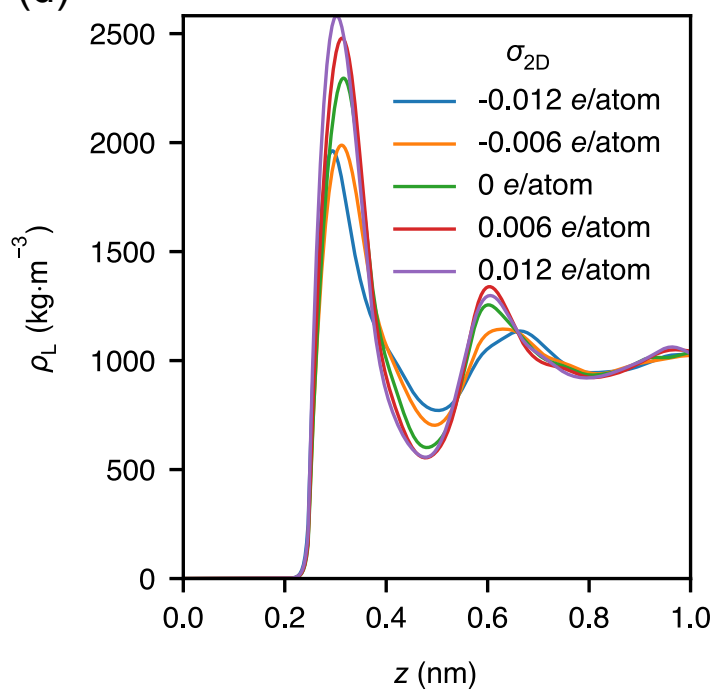

(b)

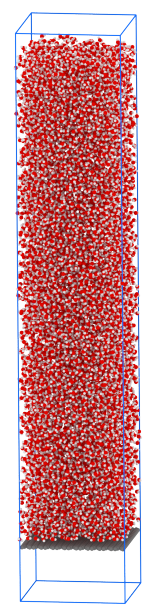

(c)

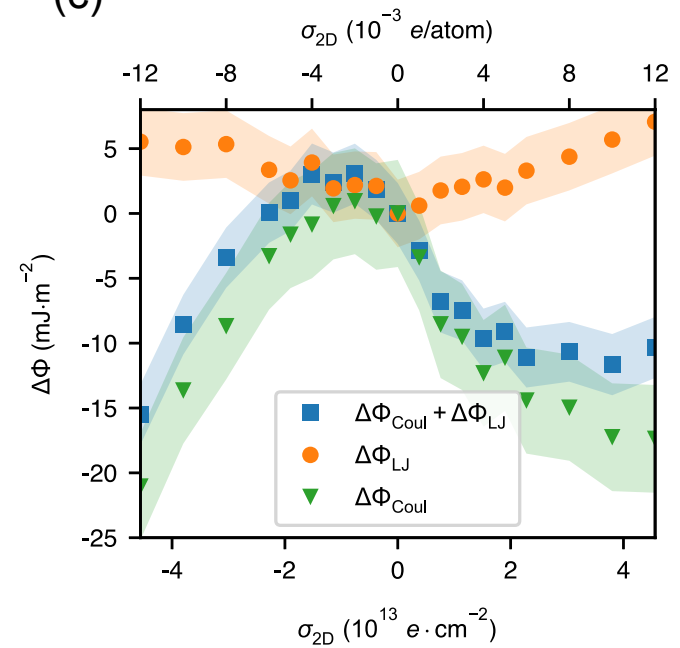

(e)

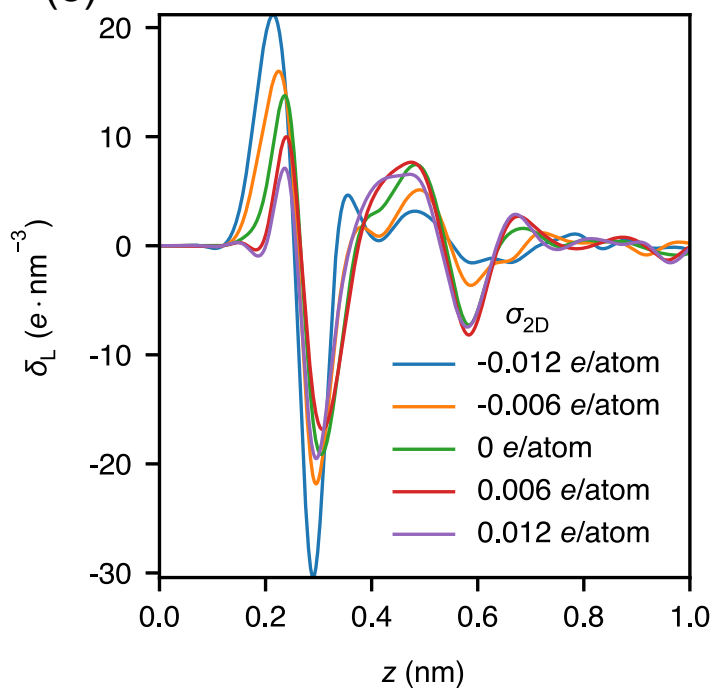

Figure 3: Geometry of the periodic MD simulation box for (a) water molecules only and (b) water-graphene systems. (c) Change of total adhesion energy $\Delta \Phi$, and its partial contributionsfrom Lennard-Jones $\Delta \Phi_{\mathrm{LJ}}$ and Coulombic $\Delta \Phi_{\text {Coul }}$ interactions, as a function of $\sigma_{2 \mathrm{D}}$. The filled regions indicate the error margin of calculation. $10^{-3}$ e/atom corresponds to a charge density of $3.80 \times 10^{12} \mathrm{e} \cdot \mathrm{cm}^{-2}$. (d) and (e) are the local density $\rho_{\mathrm{L}}$ and local charge density $\delta_{\mathrm{L}}$ of water molecules as a function of distance $z$ from graphene surface, respectively. 
(a)

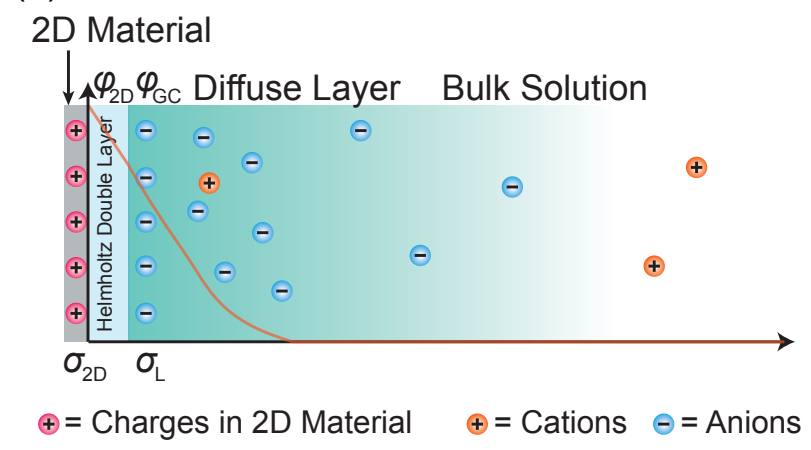

(b)
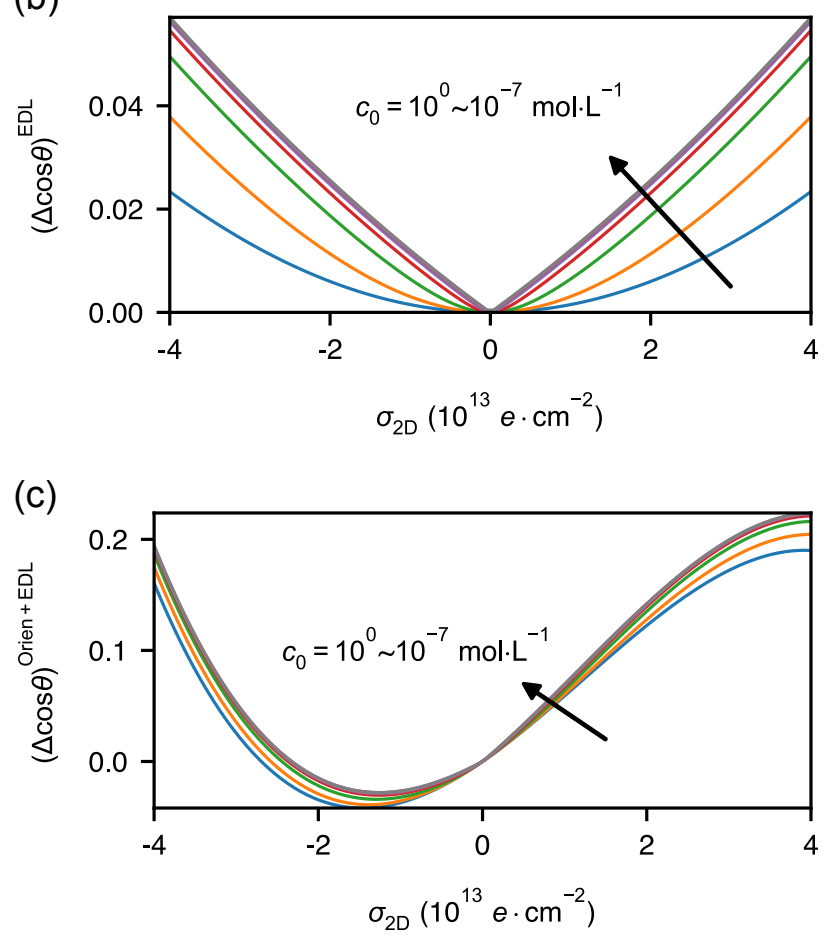

Figure 4: (a) Scheme of the interface between the 2D material and the aqueous phase. (b) $(\Delta \cos \theta)^{\mathrm{EDL}}$ as a function of $\sigma_{2 \mathrm{D}}$ with varied solute concentrations. The concentration $c_{0}$ varies from $10^{0}$ to $10^{-7} \mathrm{~mol} \cdot \mathrm{L}^{-1}$ (c) Overall change of contact angle $(\Delta \cos \theta)^{\text {Orien }+\mathrm{EDL}}$ combining the orientation and EDL effects, with varied solute concentrations as in (b). 
(a)

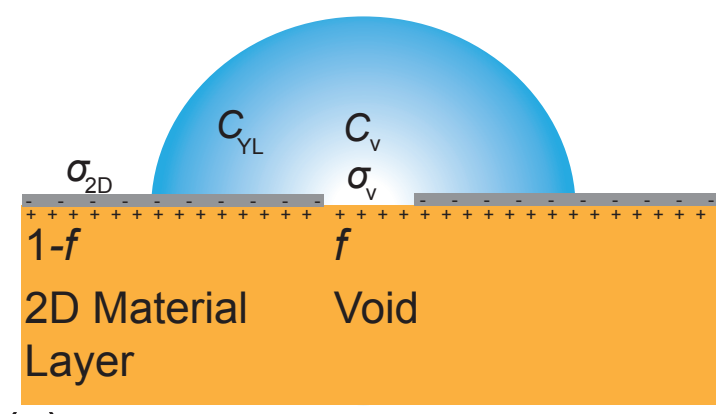

(b)

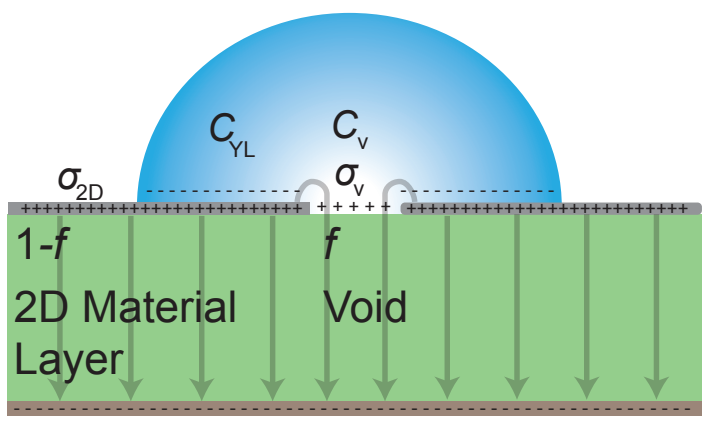

(c)

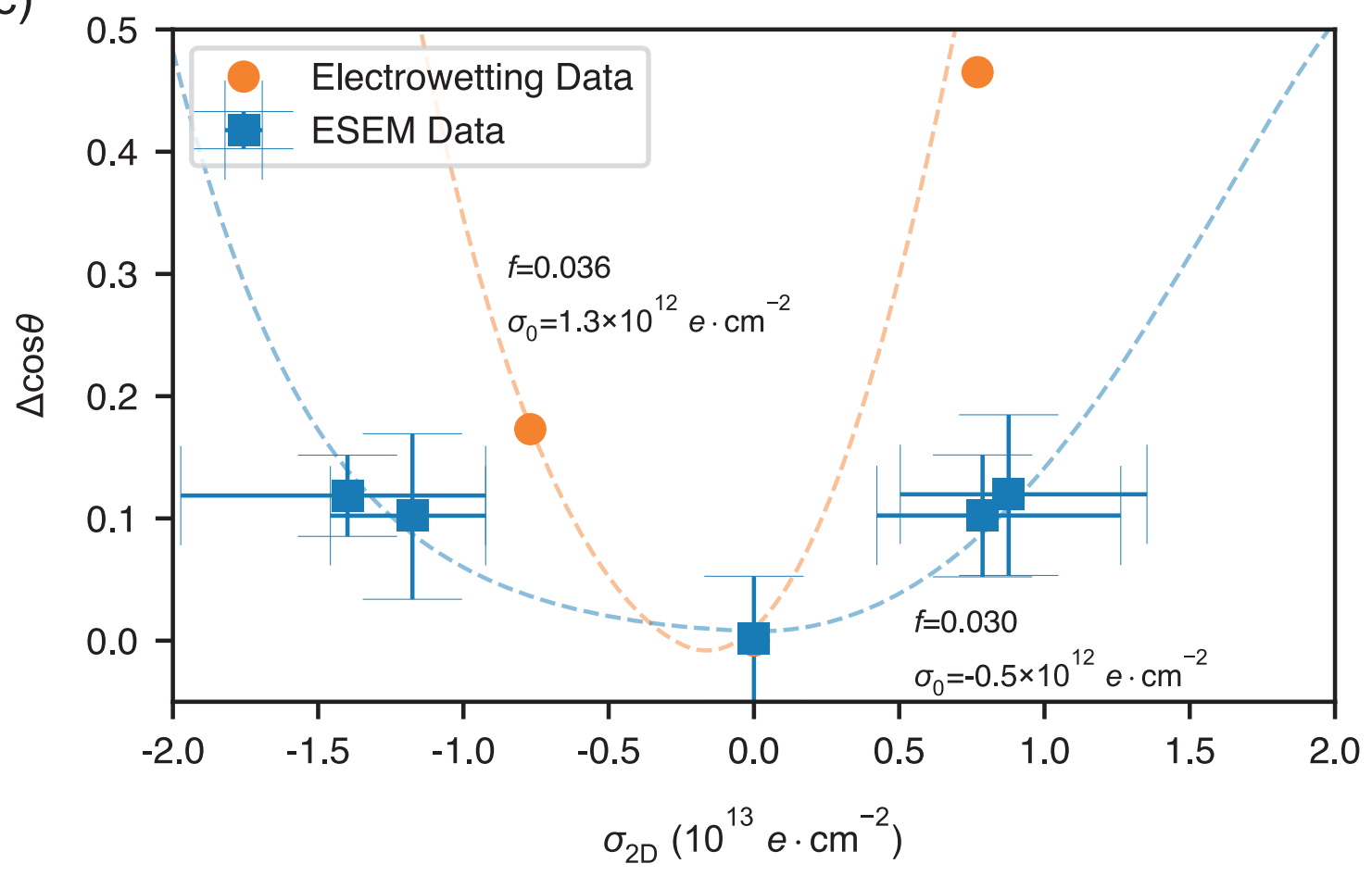

Figure 5: Schemes of the void region in (a) substrated doped 2D material and (b) electrostatic gated 2D material.(c) Fitted experimental data of $\Delta \cos \theta$ as a function of $\sigma_{2 \mathrm{D}}$. The electrowetting data are extracted from Ref. 33; the ESEM data are extracted from Ref. 35. 

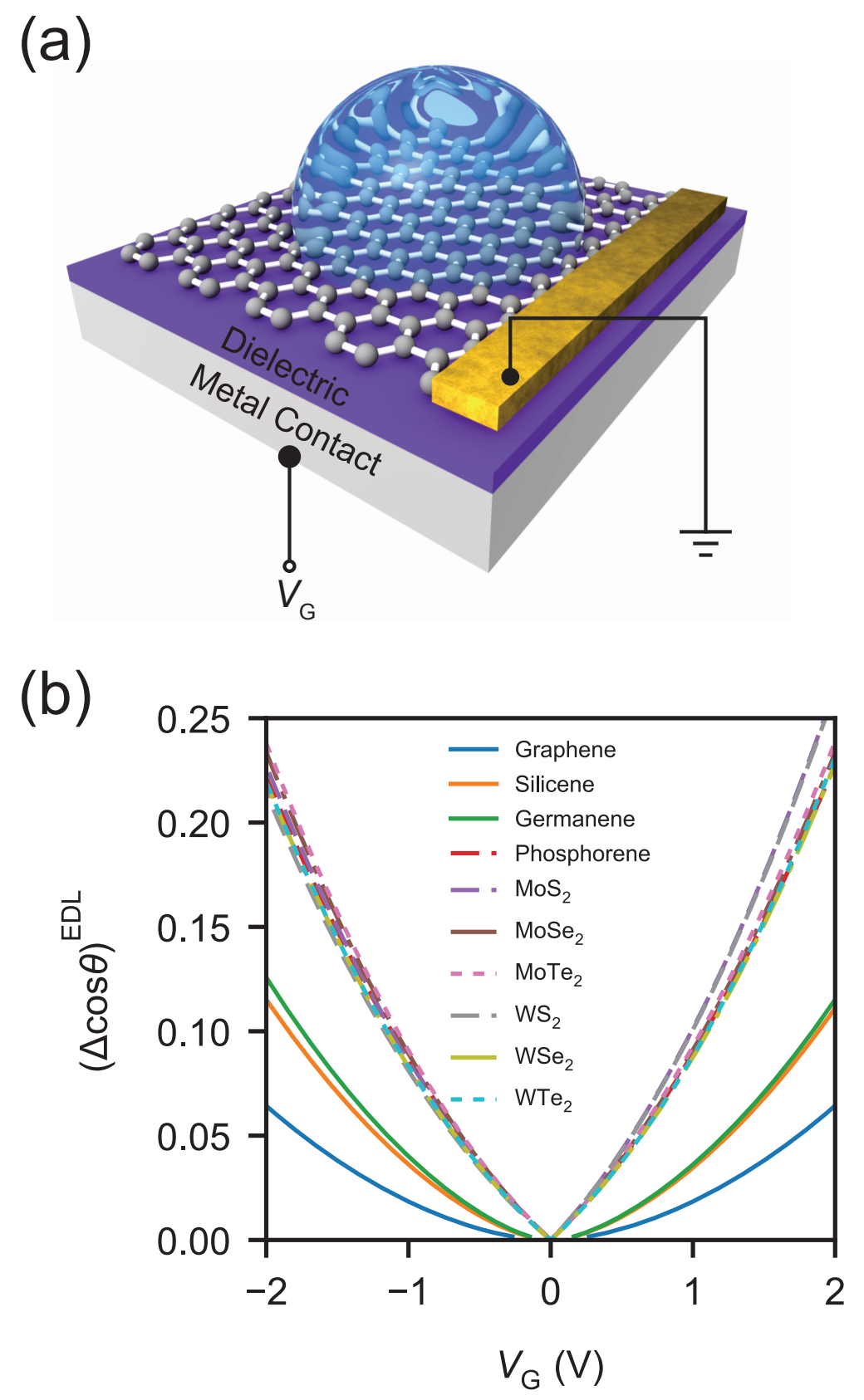

Figure 6: (a) Schematic illustration of the 2D-material-based electrowetting device, where the $2 \mathrm{D}$ material is electostatically doped. (b) $(\Delta \cos \theta)^{\mathrm{EDL}}$ as a function of $V_{\mathrm{G}}$ for selected $2 \mathrm{D}$ materials. 


\section{Graphical TOC Entry}

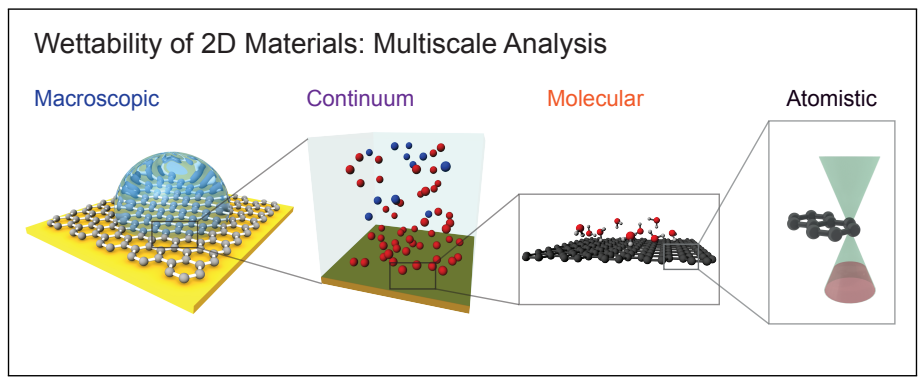

